\title{
Average intensity and scintillation of light in a turbulent biological
}

\author{
tissue \\ Hui Jina, Wei Zheng ${ }^{\mathrm{b}}$, HongTao Ma ${ }^{\mathrm{a}}$, and Yu Zhao ${ }^{\mathrm{a}, *}$ \\ ${ }^{\text {a }}$ Quality Test Center, Changchun Institute of Optics, Fine Mechanics and Physics, Chinese \\ Academy of Sciences, Changchun 130033, China \\ ${ }^{\mathrm{b}}$ Department of Ophthalmology, Affiliated Hospital of Changchun University of Traditional \\ Chinese Medicine, Changchun 130021, China
}

\begin{abstract}
Scintillation is one of the most important properties of light propagating through random media. The scintillation index of plane and spherical light waves as well as of a Gaussian beam propagating in the turbulent biological tissue is developed. Expressions of average intensity of Gaussian beams through turbulent tissue are also derived. Effects of the biological tissue turbulence on the average intensity and scintillation index are discussed in the numerical simulations. An analysis of the threshold between the weak and strong regimes of biological tissue turbulence is made with the variation of outer scale of turbulence. The results are of utmost importance for any applications involved light beam propagation through tissues, especially the cases where the average intensity and spreading properties of the light field should be taken into account to evaluate the system performance and investigations in the structures of biological tissue.
\end{abstract}

Key words: Biological tissue; Turbulence; Scintillation index; Average intensity.

\section{Introduction}

Along with the development of imaging technology in the biological tissue including optical coherence tomography (OCT) [1-3], the propagation of optical beams through a biological tissue [4-12] is a subject of considerable importance which attracted more and more researchers' attention.

As we all know, biological tissue is a very complex system in which the light is strongly scattered in propagation due to the spatial fluctuation of its refractive index. Phase-contrast microscopy shows that the structure of the refractive-index inhomogeneities in a variety of mammalian tissues resembles that of frozen turbulence, Schmitt and Kumar found that the observed structure function fits the classical Kolmogorov model of turbulence and presented the model of power spectrum refractive-index variations in biological tissue [4]. Based on this model of power spectrum of refractive-index variations in biological tissue, Gao made lots of efforts to explore the changes of coherence and the state of polarization of optical beams propagating through a biological tissue [5-9]. The amplitude of the light backscattering from living tissue is very weak. Thus to effectively measure the backscattered light from a depth beneath the surface for generating optical sectional images and obtaining characteristics of tissue, many low-coherence interferometry-based imaging techniques have been proposed [10]. Xu and Alfano utilized the fractal continuous random media to model visible and near-infrared light scattering by biological tissue and cell suspensions [11]. For an improvement of the model based on a continuous refractive index variation presented in [4], Sheppard proposed a fractal model of light scattering in biological tissue and cells based on $K$-distribution, the range of allowable power laws was extended

\footnotetext{
*731642013@qq.com
} 
into the subfractal regime [12].

The most important fourth-order statistical quantity of a wave is the scintillation index, being the normalized variance of the fluctuating intensity. The scintillation index of a plane wave, known as the Rytov variance [3], is conventionally used in atmospheric propagation for separating weak and strong turbulent regimes. As an optical beam propagates through the turbulent biological tissue, it will experience random deflections due to refractive turbulence. As a result, the average intensity of beam will decrease and the beam width will increase as a result of the effect of diffraction [13]. For stochastic electromagnetic vortex beams propagation through the turbulent biological tissues, the spectral density, the spectral degree of coherence and the spectral degree of polarization were investigated in detail [14]. The analytical formulae of anomalous hollow beams (AHBs) propagating through the turbulent biological tissues based on the extended Huygens-Fresnel integral formula and the irradiance and spreading properties of AHBs in turbulent biological tissues were studied numerically in [15]. Evidence shows that choosing the appropriate partially coherent model beam is one of the most effective methods to reduce the influence of turbulence on the laser beams propagating through the random medium [16-21].

However, to the best of our knowledge, the intensity and scintillation index of laser beams propagating through a turbulent biological tissue haven't been studied. In this paper, we put forward a model of average intensity for Gaussian beams propagating in the biological tissue turbulence on the basis of the fractal model the power spectrum of fluctuations in the refractive index in biological tissue.

The paper is organized as follows, in section 2, we derive the expressions of average intensity for Gaussian beams in turbulent biological tissue. The scintillation index model is given in section 3. The numerical simulations and analysis are given in section 4. Finally, conclusions are given in section 5.

\section{Average spectral intensity of Gaussian beams propagating through a turbulent biological tissue}

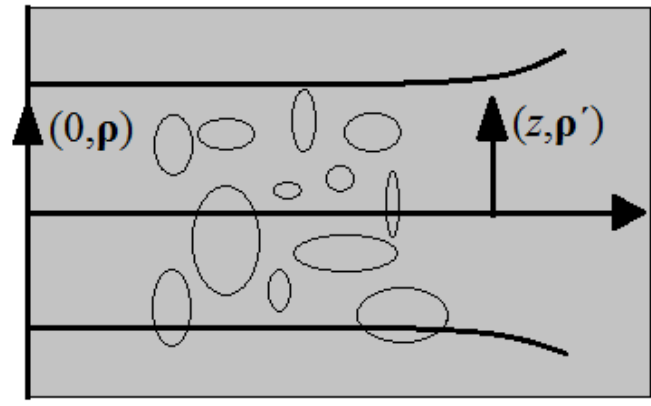

Fig. 1. Notation for a Gaussian beam propagating through a turbulent biological tissue

The cross-spectral density function (CSDF) of Gaussian random fields at the planar source surface can be written as [16]:

$$
W^{(0)}\left(\boldsymbol{\rho}_{1}, \boldsymbol{\rho}_{2} ; \omega\right)=\exp \left(-\frac{\boldsymbol{\rho}_{1}^{2}+\boldsymbol{\rho}_{2}^{2}}{4 \sigma_{s}^{2}}\right)
$$

where $\boldsymbol{\rho}_{1}$ and $\boldsymbol{\rho}_{2}$ are two-dimensional position vectors at the input plane, $\sigma_{s}$ is the rms width of the source, $\omega$ is the angular frequency.

According to the extended Huygens-Fresnel principle, upon propagation from the source plane to any plane with $z>0$ and by the paraxial approximation, the CSDF takes the form [13] 


$$
\begin{aligned}
& W\left(\boldsymbol{\rho}_{1}^{\prime}, \boldsymbol{\rho}_{2}^{\prime}, z ; \omega\right)=\frac{k^{2}}{(2 \pi z)^{2}} \iiint \int W^{(0)}\left(\boldsymbol{\rho}_{1}, \boldsymbol{\rho}_{2} ; \omega\right) \exp \left[-i k \frac{\left(\boldsymbol{\rho}_{1}^{\prime}-\boldsymbol{\rho}_{1}\right)^{2}-\left(\boldsymbol{\rho}_{2}^{\prime}-\boldsymbol{\rho}_{2}\right)^{2}}{2 z}\right] \\
& \times\left\langle\exp \left[\psi^{*}\left(\boldsymbol{\rho}_{1}, \boldsymbol{\rho}_{1}^{\prime}, z ; \omega\right)+\psi\left(\boldsymbol{\rho}_{2}, \boldsymbol{\rho}_{2}^{\prime}, z ; \omega\right)\right]\right\rangle_{a} \mathrm{~d}^{2} \rho_{1} \mathrm{~d}^{2} \rho_{2},
\end{aligned}
$$

where $\boldsymbol{\rho}_{1}^{\prime}$ and $\boldsymbol{\rho}_{2}^{\prime}$ are two-dimensional position vectors at the output plane, where $k=2 \pi n_{0} / \lambda$ is the wave number of light, with $\lambda$ being the wavelength of the source and $n_{0}$ being the background refractive index, $\psi^{*}\left(\boldsymbol{\rho}_{1}, \boldsymbol{\rho}_{1}^{\prime}, z ; \omega\right)$ represents the random part of the complex phase of aspherical wave propagating in biological tissue turbulence, the asterisk stands for the complex conjugate, and $\langle\bullet\rangle_{b}$ denotes the ensemble average of in biological tissue turbulence.

The last term in above integrand can be shown to be given by the expression $[13,20]$ :

$$
\begin{aligned}
&\left\langle\exp \left[\psi^{*}\left(\boldsymbol{\rho}_{1}, \boldsymbol{\rho}_{1}^{\prime}, z ; \omega\right)+\psi\left(\boldsymbol{\rho}_{2}, \boldsymbol{\rho}_{2}^{\prime}, z ; \omega\right)\right]\right\rangle_{b} \\
&=\exp \left\{-\frac{\pi^{2} k^{2} z}{3}\left[\left(\boldsymbol{\rho}_{1}-\boldsymbol{\rho}_{2}\right)^{2}+\left(\boldsymbol{\rho}_{1}-\boldsymbol{\rho}_{2}\right)\left(\boldsymbol{\rho}_{1}^{\prime}-\boldsymbol{\rho}_{2}^{\prime}\right)+\left(\boldsymbol{\rho}_{1}^{\prime}-\boldsymbol{\rho}_{2}^{\prime}\right)^{2}\right.\right. \\
&\left.\left.\int_{0}^{\infty} \kappa^{3} \Phi(\kappa) d \kappa\right]\right\}, \\
&= \exp \left\{-\frac{1}{\rho_{0}^{2}}\left[\left(\boldsymbol{\rho}_{1}-\boldsymbol{\rho}_{2}\right)^{2}+\left(\boldsymbol{\rho}_{1}-\boldsymbol{\rho}_{2}\right)\left(\boldsymbol{\rho}_{1}^{\prime}-\boldsymbol{\rho}_{2}^{\prime}\right)+\left(\boldsymbol{\rho}_{1}^{\prime}-\boldsymbol{\rho}_{2}^{\prime}\right)^{2}\right]\right\},
\end{aligned}
$$

where $\Phi(\kappa)$ is the power spectrum of fluctuations in the refractive index of the biological tissues,

with $\kappa<<2 k$ being spatial frequency. $\kappa$ is defined as $2 \pi /$ (eddy size), $\quad \rho_{0}$ is the coherence length of a spherical wave propagating in the turbulent biological tissue and $\Phi(\kappa)$ is given by [12]

$$
\Phi(\kappa)=\sigma^{2}\left(\frac{2}{L_{0}}\right)^{n-3 / 2} \frac{\Gamma(n)}{\Gamma(n-3 / 2)\left[1+\left(\kappa L_{0}\right)^{2}\right]^{n}},
$$

where $L_{0}$ is the outer scale of the refractive-index inhomogeneity size and in the range of $4-10 \mathrm{um}$, and $\sigma^{2}$ is defined as

$$
\sigma^{2}=\frac{\varepsilon^{2} \eta_{0} l_{\max }^{7-D_{f}}}{\left(7-D_{f}\right) \pi^{2}}
$$

where $\varepsilon^{2} \eta_{0}$ stands for a constant, $D_{f}$ represents fractal dimension, with $l_{\max }$ being the cutoff correlation length. Note that $n \geq 1.5$ is required in Eq. (8). Consider the case of a Kolmogorov fractal for $n=11 / 6, D_{f}=10 / 3$. Thus Eq. (4) can be reduced as

$$
\Phi(\kappa)=0.012 \varepsilon^{2} \eta_{0} l_{\max }^{11 / 3} L_{0}^{-4} \kappa^{-11 / 3},
$$

It is needed to mention that in the derivation of Eq. (10), $\kappa L_{0} \gg 1$ should be satisfied. According to Eqs. (3) and (6), we can obtain the coherence length of a spherical wave 
propagating in the turbulent biological tissue in Ref. [14]

$$
\left|\rho_{0}\right|=0.898\left(\frac{\varepsilon^{2} \eta_{0} l_{\max }^{3} L_{0}^{-3} n_{0} k^{2} z}{\lambda}\right)^{-1 / 2},
$$

Submitting Eqs. (6) and (7) into Eq. (4), for Gaussian beams we have

$$
\begin{aligned}
& W\left(\boldsymbol{\rho}_{1}^{\prime}, \boldsymbol{\rho}_{2}^{\prime}, z ; \omega\right)= \\
& \frac{k^{2}}{(2 \pi z)^{2}} \iiint \int \exp \left[-i k \frac{\left(\boldsymbol{\rho}_{1}^{\prime}-\boldsymbol{\rho}_{1}\right)^{2}-\left(\boldsymbol{\rho}_{2}^{\prime}-\boldsymbol{\rho}_{2}\right)^{2}}{2 z}\right] \\
& \times \exp \left\{-\frac{\left[\left(\boldsymbol{\rho}_{1}-\boldsymbol{\rho}_{2}\right)^{2}+\left(\boldsymbol{\rho}_{1}-\boldsymbol{\rho}_{2}\right)\left(\boldsymbol{\rho}_{1}^{\prime}-\boldsymbol{\rho}_{2}^{\prime}\right)+\left(\boldsymbol{\rho}_{1}^{\prime}-\boldsymbol{\rho}_{2}^{\prime}\right)^{2}\right]}{\rho_{o c}^{2}}\right\} \\
& \times \exp \left(-\frac{\boldsymbol{\rho}_{1}^{2}+\boldsymbol{\rho}_{2}^{2}}{4 \sigma_{s}^{2}}\right) \mathrm{d}^{2} \rho_{1} \mathrm{~d}^{2} \rho_{2},
\end{aligned}
$$

Then, we use the integral relation [18]:

$$
\int_{-\infty}^{+\infty} \exp \left(-a x^{2}+b x\right) d x=\frac{\sqrt{\pi}}{a} \exp \left(\frac{b^{2}}{4 a^{2}}\right),\left(\operatorname{Re}\left[a^{2}>0\right]\right),
$$

and performing the calculation, the spectral density function of a focused Gaussian beam is reduced to

$$
\begin{aligned}
& W\left(\boldsymbol{\rho}_{1}^{\prime}, \boldsymbol{\rho}_{2}^{\prime}, z ; \omega\right)= \\
& \frac{1}{A_{1}(z)} \exp \left[\frac{\left(\boldsymbol{\rho}_{1}^{\prime}+\boldsymbol{\rho}_{2}^{\prime}\right)^{2}}{8 \sigma_{s}^{2} A_{1}(z)}\right] \exp \left[-i k \frac{\left(\boldsymbol{\rho}_{2}^{\prime 2}-\boldsymbol{\rho}_{1}^{\prime 2}\right)}{2 A_{2}(z)}\right] \\
& \times \exp \left[-\left(\boldsymbol{\rho}_{1}^{\prime}-\boldsymbol{\rho}_{2}^{\prime}\right)^{2}\left(\frac{1}{2 A_{1}(z) B_{1}}+\frac{\left(1+\sigma_{s}^{2}\right)}{\rho_{0}^{2}}-\frac{z^{2}}{2 k^{2} \sigma_{s}^{2} \rho_{0}^{4} A_{1}(z)}\right)\right]
\end{aligned}
$$

where

$$
\begin{gathered}
A_{1}=1+\frac{z^{2}}{k^{2} \sigma_{s}^{2}}\left(\frac{1}{B_{1}}+\frac{2}{\rho_{0}^{2}}\right), \\
A_{2}(z)=\frac{k^{2} \sigma_{s}^{2} z A_{1}(z)}{k^{2} \sigma_{s}^{2} A_{1}(z)+z^{2} \rho_{0}^{-2}-k^{2} \sigma_{s}^{2}}, \\
B_{1}=4 \sigma_{s}^{2} .
\end{gathered}
$$

By setting $\boldsymbol{\rho}_{1}^{\prime}=\boldsymbol{\rho}_{2}^{\prime}=\mathbf{r}$, from Eq. (10), we can derive the spectral density at any point at $z$ plane [19]

$$
S(\mathbf{r}, z ; \omega)=\frac{1}{A_{1}(z)} \exp \left[-\frac{\mathbf{r}^{2}}{2 \sigma_{s}^{2} A_{1}(z)}\right] .
$$

\section{Scintillation of Gaussian beam in a turbulent biological tissue}

The scintillation index of a light wave with instantaneous intensity $I(\mathbf{r}, L, \lambda)$ at a point with position vector $(\mathbf{r}, L)=(x, y, L)$, with $L$ being the direction of propagation, is defined by the expression [9] 


$$
\sigma_{I}^{2}(\mathbf{r}, L, \lambda)=\frac{\left\langle I^{2}(\mathbf{r}, L, \lambda)\right\rangle-\langle I(\mathbf{r}, L, \lambda)\rangle^{2}}{\langle I(\mathbf{r}, L, \lambda)\rangle^{2}}
$$

where the angular brackets stand for the long-time average. Under the assumption of weakly turbulent fluctuations the scintillation index of a Gaussian beam can be represented by the sum of the longitudinal (on-axis) component $\sigma_{I, l}^{2}(0, L, \lambda)$ and the radial component $\sigma_{I, r}^{2}(\mathbf{r}, L, \lambda)$, i.e.

$$
\sigma_{I}^{2}(\mathbf{r}, L, \lambda)=\sigma_{I, l}^{2}(0, L, \lambda)+\sigma_{I, r}^{2}(\mathbf{r}, L, \lambda)
$$

where

$$
\begin{aligned}
& \sigma_{I, l}^{2}(0, L, \lambda)=8 \pi^{2} k^{2} L \int_{0}^{1} \int_{0}^{\infty} \kappa \Phi(\kappa) \exp \left[-\frac{\Lambda L \kappa^{2} \xi^{2}}{k}\right] \\
& \times\left\{1-\cos \left[\frac{L \kappa^{2}}{k} \xi(1-(1-\Theta \xi))\right]\right\} \mathrm{d} \kappa \mathrm{d} \xi, \\
& \sigma_{I, r}^{2}(\mathbf{r}, L, \lambda)=8 \pi^{2} k^{2} L \int_{0}^{1} \int_{0}^{\infty} \kappa \Phi(\kappa) \exp \left[-\frac{\Lambda L \kappa^{2} \xi^{2}}{k}\right] \\
& \quad \times\left[I_{0}(2 \Lambda r \xi \kappa)-1\right] \mathrm{d} \kappa \mathrm{d} \xi, \quad r=|\mathbf{r}| .
\end{aligned}
$$

Here $I_{0}(\cdot)$ is the modified Bessel function of order zero, $\Lambda$ and $\Theta$ are the normalized parameters of the Gaussian beam:

$$
\Lambda=\frac{\Lambda_{0}}{\Lambda_{0}+\Theta_{0}^{2}}, \quad \Theta=\frac{\Theta_{0}}{\Lambda_{0}^{2}+\Theta_{0}^{2}},
$$

with

$$
\Lambda_{0}=\frac{2 L}{k W_{0}^{2}}, \quad \Theta_{0}=1-\frac{L}{F_{0}},
$$

where $W_{0}$ and $F_{0}$ being the source plane radius and the source plane phase front radius of curvature of the Gaussian beam, submitting Eq. (6) into Eq. (17), we have

$$
\begin{aligned}
& \sigma_{I, l}^{2}(0, L, \lambda)=0.096 \varepsilon^{2} \eta_{0} l_{\max }^{3} L_{0}^{-4} \pi^{2} k^{2} L \int_{0}^{1} \int_{0}^{\infty} \kappa^{-8 / 3} \exp \left[-\frac{\Lambda L \kappa^{2} \xi^{2}}{k}\right] \\
& \times\left\{1-\cos \left[\frac{L \kappa^{2}}{k} \xi(1-(1-\Theta) \xi)\right]\right\} \mathrm{d} \kappa \mathrm{d} \xi,
\end{aligned}
$$

Using Euler's formula $\cos (x)=\operatorname{Re}[\exp (-\mathrm{i} x)]$

$$
\begin{aligned}
& \sigma_{I, l}^{2}(0, L, \lambda)=0.096 \varepsilon^{2} \eta_{0} l_{\max }^{3} L_{0}^{-4} \pi^{2} k^{2} L \operatorname{Re} \int_{0}^{1} \int_{0}^{\infty} \kappa^{-8 / 3} \mathrm{~d} \kappa \mathrm{d} \xi \\
& \left\{\exp \left[-\frac{\Lambda L \xi^{2} \kappa^{2}}{k}\right]-\exp \left[-\left(\frac{\Lambda L \xi^{2}}{k}+\mathrm{i} \frac{L \xi}{k}(1-(1-\Theta) \xi)\right) \kappa^{2}\right]\right\},
\end{aligned}
$$


For mathematical analysis convenience, we define $Q=\Lambda L / k, R=L / k$, and then

$$
\begin{aligned}
\sigma_{I, l}^{2}(0, L, \lambda) & =0.096 \varepsilon^{2} \eta_{0} l_{\max }^{3} L_{0}^{-4} \pi^{2} k^{2} L \operatorname{Re} \int_{0}^{1} \int_{0}^{\infty} \kappa^{-8 / 3} \mathrm{~d} \kappa \mathrm{d} \xi \\
& \times\left\{\exp \left(-Q \xi^{2} \kappa^{2}\right)-\exp \left[-\left(Q \xi^{2} \kappa^{2}+\mathrm{i} R \xi(1-(1-\Theta) \xi)\right) \kappa^{2}\right]\right\} .
\end{aligned}
$$

Utilizing the integral relationship in [13] and after some calculations, Eq. (23) can be reduced to

$$
\begin{aligned}
& \sigma_{I, l}^{2}(0, L, \lambda)=1.74 \varepsilon^{2} \eta_{0} l_{\max }^{3} L_{0}^{-4} \pi^{2} k^{7 / 6} L^{11 / 6} \\
& \times \operatorname{Re}\left[\mathrm{i}^{5 / 6}{ }_{2} \mathrm{~F}_{1}\left(-\frac{5}{6}, \frac{11}{6} ; \frac{17}{6} ; 1-\Theta+\mathrm{i} \Lambda\right)-\frac{11}{16} \Lambda^{5 / 6}\right]
\end{aligned}
$$

Submitting Eq. (6) into Eq. (18), we have

$$
\begin{aligned}
& \sigma_{I, r}^{2}(\mathbf{r}, L, \lambda)=0.096 \varepsilon^{2} \eta_{0} l_{\max }^{3} L_{0}^{-4} \pi^{2} k^{2} L \int_{0}^{1} \int_{0}^{\infty} \kappa^{-8 / 3} \mathrm{~d} \kappa \mathrm{d} \xi \\
& \times \exp \left[-\frac{\Lambda L \xi^{2} \kappa^{2}}{k}\right] \times\left[I_{0}(2 \Lambda r \xi \kappa)-1\right], \quad r=|\mathbf{r}| .
\end{aligned}
$$

where $I_{0}(\cdot)$ is the modified Bessel function of the first kind, and Eq. (25) can be reduced to

$$
\begin{aligned}
\sigma_{I, r}^{2}(\mathbf{r}, L, \lambda)= & 0.096 \varepsilon^{2} \eta_{0} l_{\max }^{3} L_{0}^{-4} \pi^{2} k^{2} L \sum_{n=1}^{\infty} \frac{(\Lambda r)^{2 n}}{(n !)^{2}} \int_{0}^{1} \xi^{2 n} \mathrm{~d} \xi \\
& \times \int_{0}^{\infty} \kappa^{2 n-8 / 3} \exp \left[-\frac{\Lambda L \xi^{2} \kappa^{2}}{k}\right] \mathrm{d} \kappa,
\end{aligned}
$$

Using the integral in [13],

$$
\int_{0}^{\infty} \kappa^{2 n-8 / 3} \exp \left(-a^{2} k^{2}\right) \mathrm{d} \kappa=\frac{1}{2} \frac{\Gamma(n-5 / 6)}{a^{5 / 3-2 n}}
$$

Eq. (27) can be reduced to

$$
\begin{aligned}
\sigma_{I, r}^{2}(\mathbf{r}, L, \lambda) & =0.048 \varepsilon^{2} \eta_{0} l_{\max }^{3} L_{0}^{-4} \pi^{2} k^{2} L \sum_{n=1}^{\infty} \frac{(\Lambda r)^{2 n}}{(n !)^{2}} \int_{0}^{1} \xi^{2 n} \frac{\Gamma(n-5 / 6)}{\left(\Lambda^{1 / 2} L^{1 / 2} k^{-1 / 2} \xi\right)^{5 / 3-2 n}} \mathrm{~d} \xi \\
& =0.048 \Gamma(-5 / 6) \eta_{0} l_{\max }^{3} L_{0}^{-4} \pi^{2} k^{7 / 6} L^{11 / 6} \Lambda^{5 / 6}\left[\sum_{n=1}^{\infty} \frac{(-5 / 6)_{n}}{(1)_{n} n !}\left(\frac{2 r^{2}}{W^{2}}\right)^{n}-1\right] \\
& =1.18 \eta_{0} l_{\max }^{3} L_{0}^{-4} \pi^{2} k^{7 / 6} L^{11 / 6} \Lambda^{5 / 6}\left[1-{ }_{1} \mathrm{~F}_{1}\left(-\frac{5}{6} ; 1 ; \frac{2 r^{2}}{W^{2}}\right)\right]
\end{aligned}
$$

In practice, it is generally useful to have simple analytic approximations for the scintillation index. Accurate approximations covering general Gaussian beam waves have been developed for several spectral models, for Kolmogorov fractal spectrum, an approximation [13] for Eq. (28) is 


$$
\sigma_{I, r}^{2}(\mathbf{r}, L, \lambda)=1.98 \eta_{0} l_{\max }^{3} L_{0}^{-4} \pi^{2} k^{7 / 6} L^{11 / 6} \Lambda^{5 / 6} \frac{2 r^{2}}{W_{0}^{2}\left(\Theta_{0}^{2}+\Lambda_{0}^{2}\right)}
$$

Coupled with an approximation for the longitudinal term in the case of a collimated or divergent beam, the total scintillation index cab be closely approximated [13] by the simple algebraic form, we can obtain the expression of the scintillation index of Gaussian beam propagating through a turbulent biological tissue:

$$
\begin{aligned}
& \sigma_{I}^{2}(\mathbf{r}, L, \lambda)=1.74 \varepsilon^{2} \eta_{0} l_{\max }^{3} L_{0}^{-4} \pi^{2} k^{7 / 6} L^{11 / 6} \\
& \times\left\{\frac{2}{5}\left[(1+2 \Theta)^{2}+4 \Lambda^{2}\right]^{5 / 12} \cos \left[\frac{5}{6} \tan ^{-1}\left(\frac{1+2 \Theta}{2 \Lambda}\right)\right]-\frac{11}{16} \Lambda^{5 / 6}\right\} \\
& +1.98 \eta_{0} l_{\max }^{3} L_{0}^{-4} \pi^{2} k^{7 / 6} L^{11 / 6} \Lambda^{5 / 6} \frac{2 r^{2}}{W_{0}^{2}\left(\Theta_{0}^{2}+\Lambda_{0}^{2}\right)}, r<W_{0} \sqrt{\Theta_{0}^{2}+\Lambda_{0}^{2}} .
\end{aligned}
$$

\section{Numerical simulations and analysis}

In this section, we present some numerical results and discussions about the statistical properties of the stochastic electromagnetic vortex beams through the turbulent biological tissues are presented in the following. The mouse liver tissue is taken for example and the correlated data in Ref. [8], $l_{\max }=2.3 \mu m, L=1.4 \mu m$, $\varepsilon^{2} \eta_{0}=9.854 \times 10^{-4}, \lambda=0.83 \mu \mathrm{m}$ are employed in our simulations, unless other values are indicated in the figures and illustrations.
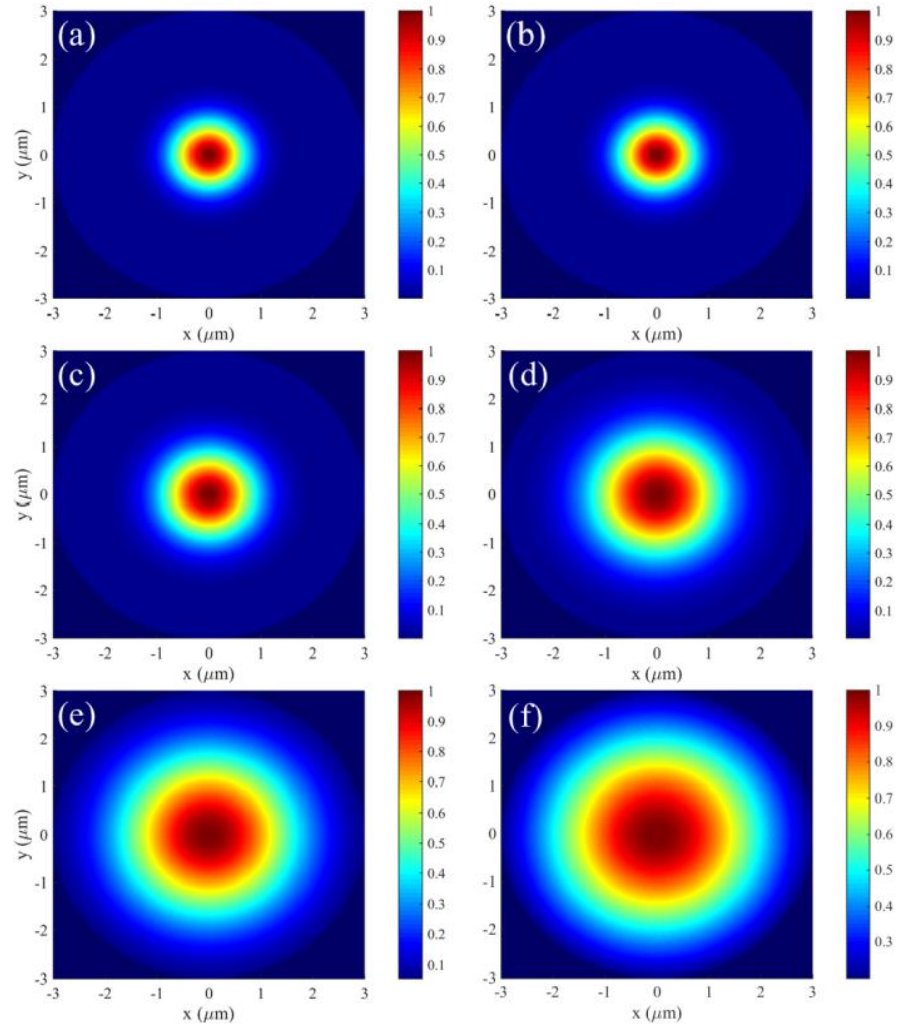

Fig. 2. Normalized average intensity of GSM beams propagating through a turbulent biological tissue versus different propagation distance at $\mathrm{z}=0$ (a), $\mathrm{z}=100 \mu \mathrm{m}$ (b) $\mathrm{z}=200 \mu \mathrm{m}$ (c), $\mathrm{z}=300 \mu \mathrm{m}$ (d), $\mathrm{z}=400 \mu \mathrm{m}$ (e) and $\mathrm{z}=500 \mu \mathrm{m}$ (f). 
Fig. 2 displays the normalized average intensity for Gaussian beams propagating through turbulent biological tissue at different propagation distance $z$ plane. As indicated by Fig. 2, as the beam propagates, the central peak submerges (Gaussian-distribution like), the Gaussian beams keeps the Gaussian-distribution profile all the time and spreading as the propagation distance increasing. Fig. 3 plots that the average intensity versus different the outer scale of the fractal behavior. As indicated by Fig. 3, when the propagation distance is fixed, the average intensity Gaussian beams increases as $L$ increasing. This is as a result of larger value of $L$ will lead to a stronger effect of refraction, the smaller value of $L$ will lead to an effect of stronger scattering, thus the average intensity GSM beams increases as $L$ increasing and the spreading of GSM beams decrease as the increasing $L$.
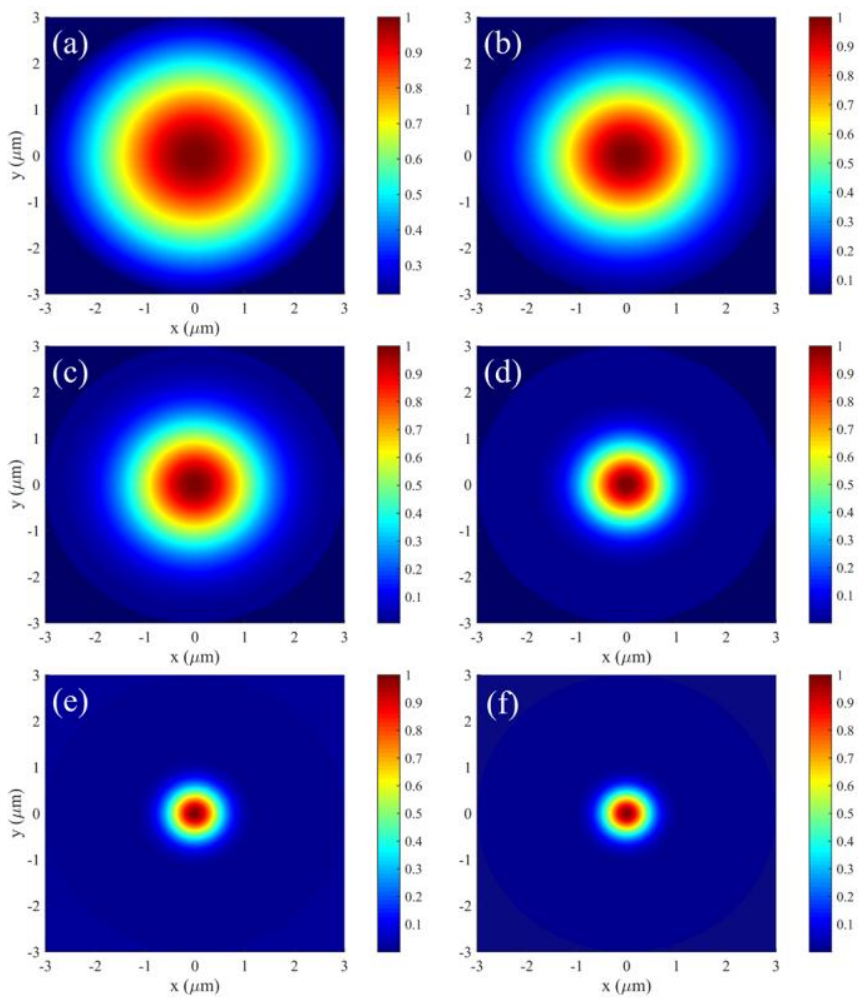

Fig. 3. Normalized average intensity of Gaussian beams propagating through a turbulent biological tissue versus different the outer scale of the fractal behavior for $L_{0}=0.8 \mu \mathrm{m}$ (a), $L_{0}=1.2 \mu \mathrm{m}$ (b) $L_{0}=1.6 \mu \mathrm{m}$ (c), $L_{0}=2.0 \mu \mathrm{m}(\mathrm{d}), L_{0}=2.2 \mu \mathrm{m}(\mathrm{e}), L_{0}=2.4 \mu \mathrm{m}$ (f) at z=50 $\mu \mathrm{m}$ plane.

On the basis of Eq. (8), one can determine the scintillation index of a Gaussian beam propagating through the turbulent biological tissue. We will first examine the special cases of a plane wave $(\Lambda=0, \Theta=1)$ and a spherical wave $(\Lambda=0, \Theta=0)$. In these two cases the radial component of the scintillation index vanishes. This can be the threshold for the propagation distances where the separation between weak and strong turbulence lies. In Fig. 4 the scintillation index of a plane wave and a spherical wave are presented as a function of propagation distance $L$ from the source plane for several values of the outer scale of the fractal behavior $L_{0}$. The scintillation levels for these waves differ by approximately one order of magnitude, which is a larger discrepancy compared with atmospheric propagation ( 0.4 for the Kolmogorov power spectrum). The scintillation index of a plane wave in atmospheric propagation is called the Rytov variance $\sigma_{R}^{2}$ and is used as a threshold for separating the weak and strong regimes of turbulent fluctuations: $\sigma_{R}^{2}<<1$ for weak conditions and $\sigma_{R}^{2}>>1$ for strong conditions. In Fig. 4(a) we show the onset of strong fluctuations for biological tissue propagation. Depending on $L_{0}$ it can occur at distances as short 
as $130 \mu \mathrm{m}$ and as long as $300 \mu \mathrm{m}$. As seen from the curves in Fig. 4(a) and (b), the scintillation index decreases as the increasing the outer scale of the fractal behavior $L_{0}$ when the beam propagates through the biological turbulent tissue. Fig. 5 plots the scintillation index as a function of propagation distance $L$ from the source plane for several different values of the wavelength $\lambda$, as indicated by Fig. 5, the scintillation index spherical wave decreases as the increasing of wavelength $\lambda$, as we know that beams spread more rapidly with lager wavelength, thus the contribution of diffraction effect to scintillation is greater to the effect of refraction effect, hence beams with larger wavelength will lead to a lower scintillation index.
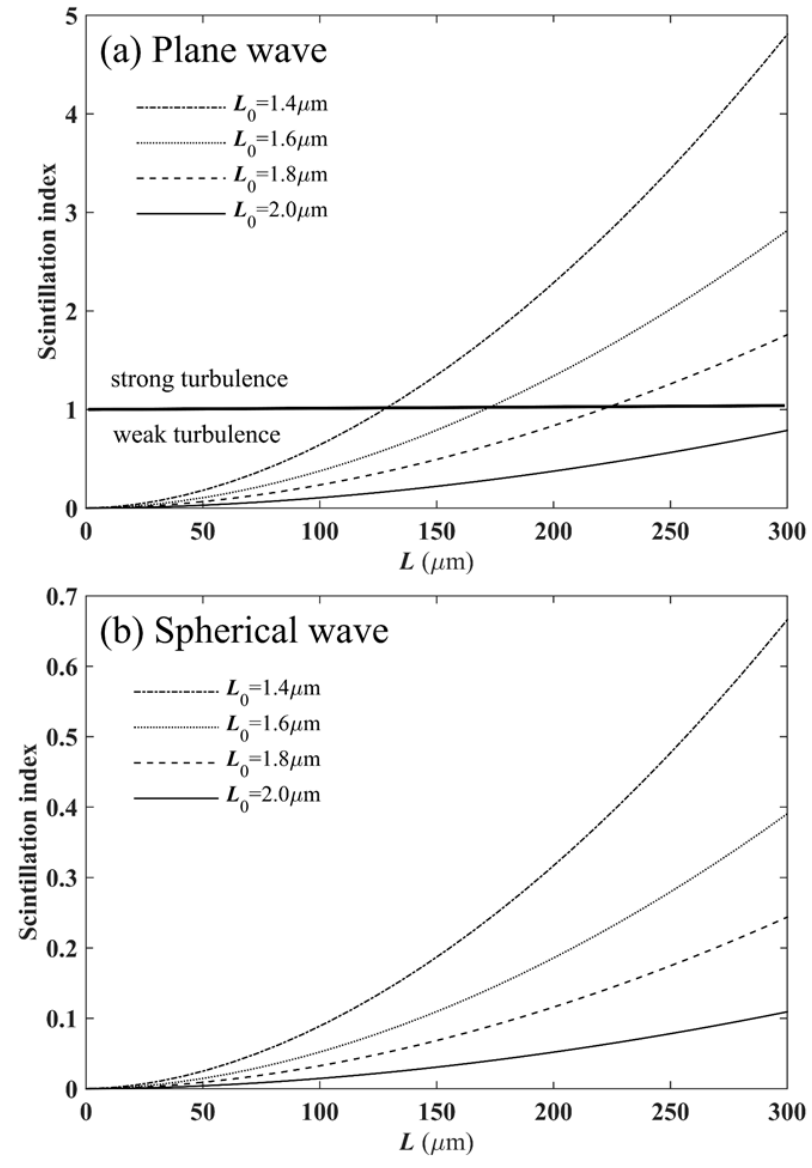

Fig. 4. The scintillation index of a plane wave (a) and a spherical wave (b) versus propagation distance $L$ [ $\mu \mathrm{m}]$ for different the outer scale of the fractal behavior $L_{0}$.

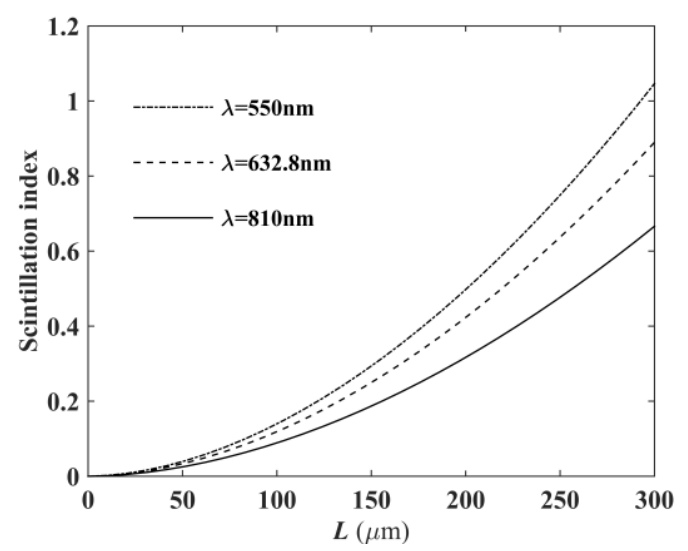

Fig. 5. The scintillation index of a spherical wave versus propagation distance $L[\mu \mathrm{m}]$ for different values of wavelength $\lambda$. 


\section{Conclusion}

In summary, we have developed the model of scintillation index of plane and spherical light waves as well as of a Gaussian beam propagating in the turbulent biological tissue. Expressions of average intensity of Gaussian beams through a turbulent tissue are also derived. Our simulation results indicate that the normalized intensity of Gaussian beam increases as the increasing propagation distance and the Gaussian beams keeps the Gaussian-distribution profile all the time, the normalized intensity decreases as the increasing outer scale of the fractal behavior $L_{0}$. An analysis of the threshold between the weak and strong regimes of biological tissue turbulence is made with the variation of outer scale of turbulence. The onset of strong fluctuations for biological tissue propagation depending on $L_{0}$ it can occur at distances as short as $130 \mu \mathrm{m}$ and as long as $300 \mu \mathrm{m}$. The scintillation index decreases as the increasing the outer scale of the fractal behavior $L_{0}$ and the increasing wavelength when the beam propagates through the biological turbulent tissue. Our results obtained in our paper can be helpful for any applications involved light beam propagation through tissues, especially the cases where the average intensity and spreading properties of the light field should be taken into account to evaluate the system performance and investigations in the structures of biological tissue.

\section{ACKNOWLEGMENTS}

This work is supported by National Science Foundation of China (Grant Nos. 61307114, 1263236, 0968895, 1102301) and the 863 Program (2013AA014402)

\section{References}

1. Huang D, Swanson E A, Lin C P, Schuman J S ,Stinson W G, Chang W, et al. "Optical coherence tomography," Science 254, 1178-1181 (1991).

2. Fercher A F, Hitzenberger C K, Kamp G, El-Zaiat S. Y., "Measurement of intraocular distances by backscattering spectral interferometry. Opt Commun. 117, 43-48 (1995).

3. Häusler G, Lindner M W. Coherence radar and spectral radar-new tools for dermatological diagnosis. J. Biomed. Opt. 3, 21-31 (1998).

4. J. M. Schmitt and G. Kumar, "Turbulent nature of refractive-index variations in biological tissue," Opt. Lett. 21(16), 1310-1312 (1996).

5. W. Gao, "Spectral changes of the light produced by scattering from tissue," Opt. Lett. 35, 862-864 (2010).

6. W. Gao, "Changes of polarization of light beams on propagation through tissue," Opt. Commun. 260, 749-754 (2006).

7. W. Gao and O. Korotkova, "Changes in the state of polarization of a random electromagnetic beam propagating through tissue," Opt. Commun. 270, 474-478 (2007).

8. W. Gao, "Effects of different correlations of partially coherent electro-magnetic beam on three-dimensional spectral intensity distribution in the focal region," Opt. Commun. 283, 4572-4581 (2010).

9. W. Gao, "Effects of the coherence and the vector properties of the light on the resolution limit in the stimulated emission depletion fluorescent microscopy. J. Opt. Soc. Am. A 25, 1378-1382 (2008).

10. W. Gao, "Change of coherence of light produced by tissue turbulence," Journal of Quantitative Spectroscopy \& Radiative Transfer 131(12), 52-58 (2013).

11. M. Xu and R. R. Alfano, "Fractal mechanisms of light scattering in biological tissue and cells," Opt. Lett. 30(22), 3051-3053 (2005).

12. Colin J. R. Sheppard, "Fractal model of light scattering in biological tissue and cells," Opt. Lett. 32(2), 142-144 (2007).

13. L. C. Andrews and R. L. Phillips, Laser Beam Propagation through Random Media, 2nd ed. (SPIE press, Bellingham, WA, 2005).

14. M. Luo, Q. Chen, L. Hua, and D. Zhao, "Propagation of stochastic electromagnetic vortex beams through the turbulent biological tissues," Phys. Lett. A 378(3), 308-314 (2014).

15. X. Lu, X. Zhu, K. Wang, C. Zhao and Y. Cai, "Effects of biological tissues on the propagation properties of anomalous hollow beams." Optik 127(4), 1842-1847 (2016).

16. Z. Mei and O. Korotkova, "Random sources generating ring-shaped beams," Opt. Lett. 38(2), 91-93(2013).

17. V. L. Minorov and V. V. Nosov, "On the theory of spatially limited light beam displacement in a randomly inhomogeneous medium," J. Opt. Soc. Am. 67, 1073-1080 (1977).

18. I. S. Gradshteyn and I. M. Ryzhik, Table of Integrals-Series-and Products, (Academic Press, Burlington, 2007). 
19. O. Korotkova, S. Sahin, and E. Shchepakina, "Multi-Gaussian Schell-model beams," J. Opt. Soc. Am. A 29(10), 2159-2164 (2012).

20. Y. Wu, Y. Zhang, Y. Li, and Z. Hu, "Beam wander of Gaussian-Schell model beams propagating through oceanic turbulence," Opt. Commun. 371, 59-66 (2016).

21. H. T. Eyyuboglu, "Propagation of higher order Bessel-Gaussian beams in turbulence," Appl. Phys. B 88(2), 259-265 (2007). 